\title{
Study on Structure and Properties of New Heterocyclic Aramid Fibers
}

\author{
Yingdeng Song ${ }^{\mathrm{a}}$, Bintai $\mathrm{Li}^{\mathrm{b}}$ and Liying $\mathrm{Xing}^{\mathrm{c}}$ *
}

Beijing Institute of Aeronautical Materials, Science and Technology on Advanced composite

Laboratory, AVIC Composite Center, Beijing 100095, China.

aasongyingdeng@163.com, blibt@avic.com, vcd4321@sina.com

Corresponding Author: Liying Xing

Keywords: heterocyclic aramid fiber, Kevlar-49, structure-property relation, wide-angle X-ray diffraction

\begin{abstract}
The structure parameters of a new heterocyclic aramid fiber (F-3B) and the Kevlar-49 fiber were characterized using wide-angle X-ray diffraction (WAXD) in detail. The tensile strength and modulus of aramid fibers were determined by monofilament tensile experiment. And the correlation of structure parameters with mechanical properties of aramid fibers was investigated. The results show that F-3B has higher tensile modulus and strength than Kevlar-49 due to its more perfect and higher orientation of crystallites in the fiber axial direction.
\end{abstract}

\section{Introduction}

The poly (para-phenylene terephthalamide) (PPTA) fibers have been studied extensively due to their application in a wide range of fiber reinforced composites, cables and ballistic fabrics et al [1]. Their excellent properties continue to draw interest in the area of structure-property relationships [2-4]. The heterocyclic aramid fiber, for example Armos, has higher tensile properties than PPTA fibers [5]. There is a pronounced difference in structure of PPTA and heterocyclic aramid fibers. The macromolecule chains of heterocyclic aramid fiber contain phenylbenzimidazole groups and its chemistry structure can be seen as a kind of modified PPTA. The structure of heterocyclic aramid fibers has been investigated by some authors [6,7]. Zavadskii and coworkers suggested a high degree of organization in the Armos fiber axial direction [8]. Wang and coworkers have compared the difference of crystallinity and properties of heterocyclic aramid fibers and Kevlar-49 [9]. From the above it is clear that different workers have focused on specific structure parameters exclusively without due consideration for other structural parameters for aramid fibers. Furthermore, the heterocyclic aramid fiber used in this paper is different from Armos which is little crystallinity [1]. Therefore, in this contribution, the properties and structure of new heterocyclic aramid fibers were carefully measured and most relative structure parameters were investigated.

\section{Experimental details}

Two kinds of aramid fibers were investigated in this work. Kevlar-49 fiber was supplied by DuPont, and the new heterocyclic aramid fiber (F-3B) was provided by China Blue star Chengrand Chemical Co. Ltd. The chemical structures of Kevlar-49 and F-3B are showed in Fig.1.

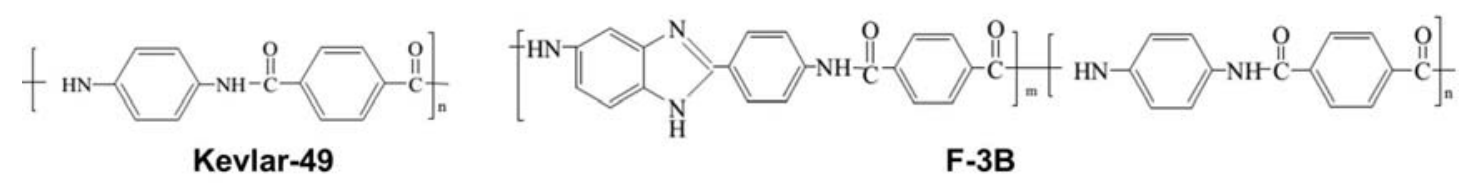

Fig.1 Chemical structures of Kevlar-49 and F-3B.

Structural characterization was carried out using wide-angle X-ray diffraction (WAXD). The structure parameters investigated include paracrystalline parameter, $g_{I I}$; equatorial X-ray diffraction crystallinity, $X$; apparent crystal sizes measured from (110), (200), and (002) planes, $L_{h k l}$; and Hermans orientation factor of $(006), f_{006}$. The structure parameters were obtained using Ni filtered $\mathrm{CuK} \alpha$ radiation and a PANalytical X'Pert PRO X-ray diffractometer in symmetric transmission 
geometry at room temperature. The apparent crystal sizes from the (110) and (200) reflections and the equatorial crystallinity were obtained from the equatorial scan. The scanning range of $2 \theta$ was from 5 to $50^{\circ}$. After corrections, the intensity profile was resolved into three peaks, 110,200 , and 211 , and an amorphous component. Voigt functions were assumed for these peaks. Then, the full width at half maximum intensity (FWHM) and the integrated intensity of peaks were calculated. The apparent crystal sizes were calculated using the Scherrer equation. And the equatorial crystallinity is defined as $X=\sum I_{i} /\left(\sum I_{i}+I_{a}\right)$, where $I_{i}$ is the integrated intensity of a diffraction peak and $I_{a}$ the integrated intensity of the amorphous component. The paracrystalline parameter and apparent crystal sizes were obtained from the meridional scan [3]. The Hermans orientation factor of (006) was obtained from the azimuthal angle scans of (006).

Tensile modulus and strength were determined by mounting individual aramid fibers on paper cards using a slow-setting epoxy resin cured at room temperature. The fiber diameters were measured using scan electron microscope (SEM). The paper cards were mounted on Instron 4445, stress-strain curves of the fibers were obtained using a crosshead speed of $2 \mathrm{~mm} / \mathrm{min}$ and a $5 \mathrm{~N}$ capacity load cell. For different gauge length 50 specimens were employed, and the average modulus values were extrapolated to infinite gauge length to account for end effects.

\section{Results and discussion}

\subsection{Structure parameters}

The equatorial and meridional diffractometer scans of Kevlar-49 and F-3B are shown in Fig.2. The significant difference in equatorial and meridional diffraction curves indicates the pronounced anisotropy of these fibers. Kevlar fiber, whose structure has been studied in the most detail [10], was used as the comparison sample. The data in Fig. 2a show that a set of sharp diffraction reflexes on the equatorial scattering curve is characteristic of Kevlar-49 fiber. In addition, the monotonic increase in diffraction intensity on the equator in $15-19^{\circ}$ region of $2 \theta$ angles and the smooth decrease in tht $2 \theta$ region of $25-35^{\circ}$ indicates the presence of a diffuse halo for this polymer. Therefore, Kevlar- 49 fiber contains both amorphous regions and segments having three-dimensional long-range order in the position of the molecular chains, which is in agreement with the published data [11]. The X-ray diffraction analysis shows that a sharp peak at $2 \theta$ of $20.2^{\circ}$ and a small shoulder peak at $2 \theta$ of $21.3^{\circ}$ and the smooth decrease in the $2 \theta$ region of $22-32^{\circ}$ appear on the equatorial scattering curves (Fig.2a) for F-3B fiber, indicating the absence of rigorous order in the position of the molecular chains in the direction perpendicular to the fiber axis. Meridional scans for these fibers (Fig.2b) is characterized by intense narrow diffraction reflections. It is shown that F-3B has more sharp peak profiles than Kevlar-49. This indicates F-3B has higher degree of organization in longitudinal direction than Kevlar-49.
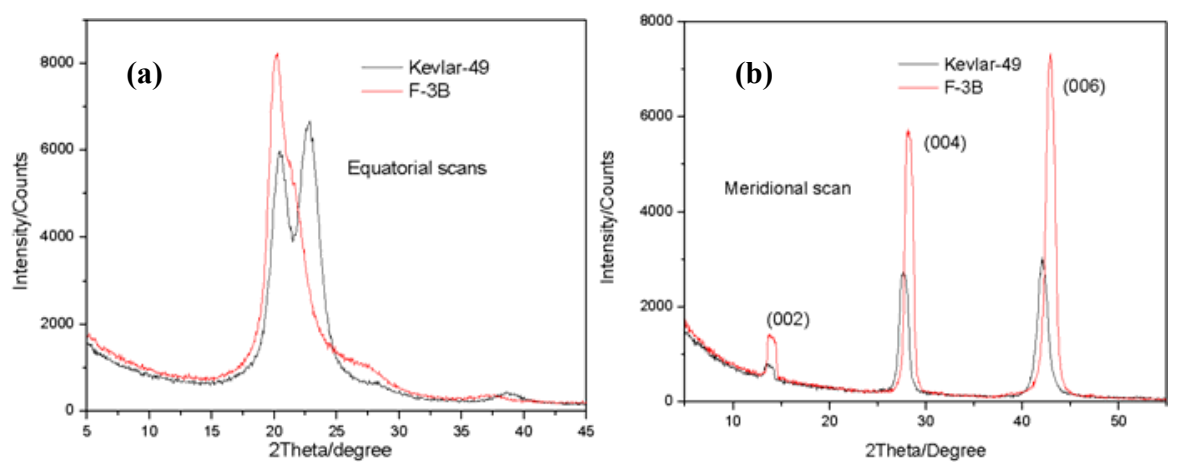

Fig.2 Curves of equatorial (a) and meridional (b) scans of Kevlar-49 and F-3B.

The peak fitting profiles of equatorial scans of Kevlar-49 and F-3B are shown in Fig.3a and Fig.3b, respectively. The results of crystallinity, $X$, and apparent crystal sizes calculated from Fig.3a and Fig. $3 \mathrm{~b}$ are listed in Table 1. The Hermans orientation factor of $(006), f_{006}$, is used to characterized the degree of organization of crystallite in longitudinal direction, according the azimuthal angle scan profile shown in Fig.4a. And the results of $f_{006}$ are shown in Table 1. Fig. $4 \mathrm{~b}$ shows a plot of the square of the meridional half peak broadening (FWHM) as a function of the fourth power of the order. There 
is a linear relationship between the two quantities, indicating that the scattering is consistent with a one-dimensional paracrystalline structure [11]. The results of paracrystalline parameter, $g_{I I}$, determined from Fig. $4 \mathrm{~b}$ are shown in Table 1, too.
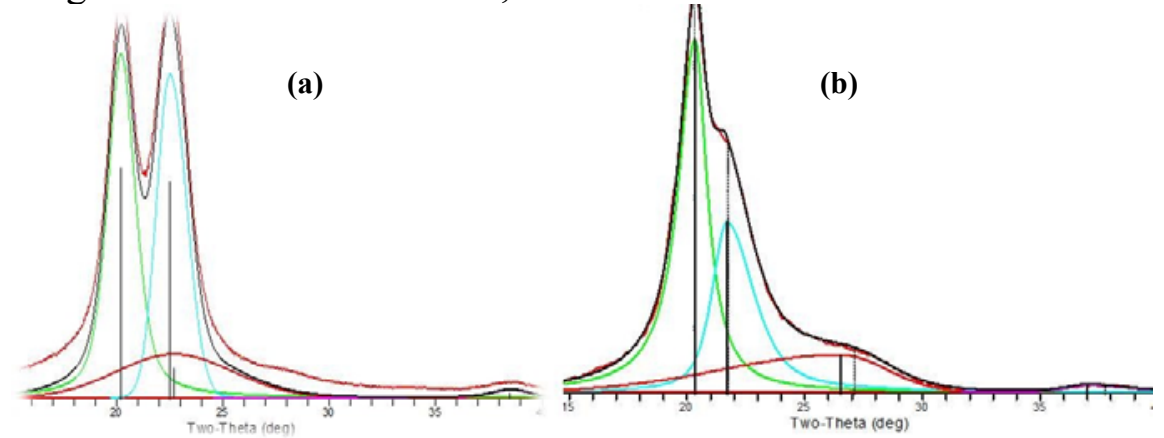

Fig.3 Peak fit profiles of Kevlar-49 (a) and F-3B (b).
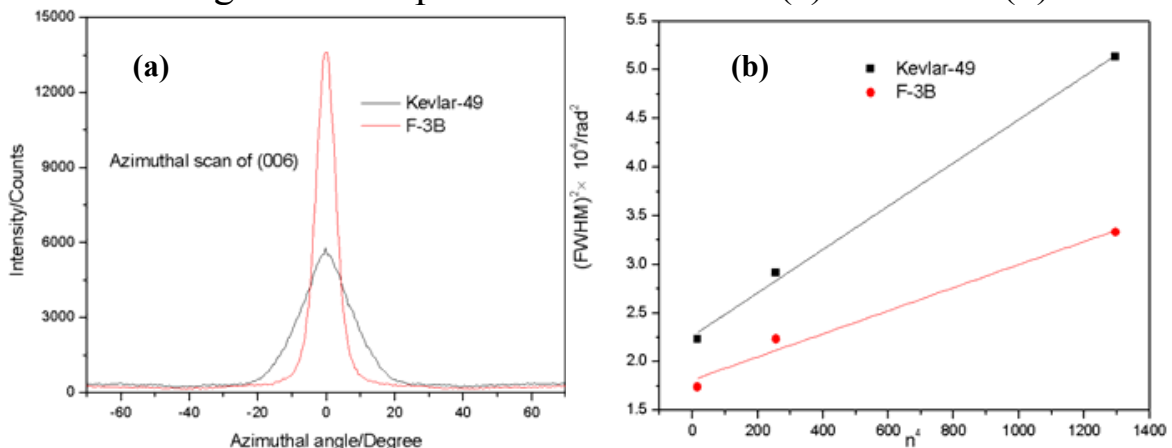

Fig.4 Curves of (a) azimuthal angle scans and (b) profile broadening analysis of meridional reflections of aramid fibers.

Table 1 The tensile properties and structure parameters of different aramid fibers.

\begin{tabular}{|c|c|c|c|c|c|c|c|c|}
\hline \multirow[t]{2}{*}{ Fiber } & \multicolumn{3}{|c|}{$\begin{array}{l}\text { Apparent crystal } \\
\text { size/nm }\end{array}$} & \multirow[t]{2}{*}{$X / \%$} & \multirow{2}{*}{$\begin{array}{l}g_{I I} \\
1 \%\end{array}$} & \multirow{2}{*}{$f_{006}$} & \multirow{2}{*}{$\begin{array}{l}\text { Strength } \\
\text { /GPa }\end{array}$} & \multirow{2}{*}{$\begin{array}{l}\text { Modulus } \\
\text { /GPa }\end{array}$} \\
\hline & $L_{110}$ & $L_{200}$ & $L_{002}$ & & & & & \\
\hline Kevlar-4 & 5.1 & 4.9 & 9.6 & 83.95 & 2.48 & 0.9516 & 3.04 & 111 \\
\hline F-3B & 5.9 & 4.3 & 10.7 & 75.88 & 2.10 & 0.9884 & 4.36 & 158 \\
\hline
\end{tabular}

\subsection{Structure and property}

Table 1 compares the mechanical properties and structure parameters of different aramid fibers. It is shown that the tensile strength and modulus of F-3B is higher than Kevalr-49. The table clearly suggests that the structure parameters determine the difference in mechanical properties. As can be seen in the table, the apparent crystal size, $L_{110}$ and $L_{002}$, and crystallinity of Kevlar-49 are higher than F-3B. However, the $L_{200}, f_{006}$, and $g_{I I}$ of Kevlar- 49 are lower than F-3B. In addition, the tensile strength and modulus of Kevlar-49 are lower than F-3B. It was confirmed that the structure parameters need to be considered to explain the difference of tensile properties.

There is a pronounced difference in structure of Kevlar-49 and F-3B shown in Fig.1. The macromolecule chains of heterocyclic aramid fiber contain phenylbenzimidazole (PHA) groups, and its chemistry structure can be seen as a kind of modified PPTA. The volume of heterocyclic structure, PHA, is larger than PPTA unit in a macromolecule chain. Thus the crystal size of (110) of F-3B is enlarged for steric reason. Furthermore, the PHA structure changes the dry-jet wet spinning process by which Kevlar-49 is made. The absence of liquid crystalline domains in spinning solution makes it possible to regulate structure building at the fiber forming and thermal treatment stages to give maximal orientation order. Owing to the lack of a plane of symmetry in the heterocyclic groups and to the mixed linking of monomers, the extended chain conformations are irregular and lead to lower crystalline order, with a consequent reduction in the possibility of axial movement. Therefore, the crystallinity of F-3B is lower, but higher than Armos reported in [1]. However, the less regular 
molecular chain structure leads to a higher proportion of stress-holding molecular chains and therefore to mechanical properties that are superior in F-3B to Kevlar-49. Moreover, the distance of adjacent chains in perpendicular to (200) direction, $L_{200}$, is reduced for F-3B. That means the crystal structure has a more perfect 2 symmetry in F-3B. This also promotes the formation of hydrogen bonds between chains within the $b c$ plane [3]. Therefore the number of hydrogen bonds increases in the crystallite of F-3B. In addition, the higher $L_{002}$ and lower $g_{I I}$ of F-3B show that the crystallite of F-3B is more perfect in longitudinal direction. Moreover, $f_{006}$ of F-3B is higher than Kevlar-49, indicating that the crystallite of F-3B has higher degree of orientation in longitudinal direction than Kevlar-49.

Accroding to the above, the tensile strength and modulus of F-3B are higher than Kevlar-49, which is due to combination of more perfect and higher orientation of crystallites in the longitudinal direction of F-3B fiber.

\section{Summary}

The structure parameters and tensile properties of a new heterocyclic aramid fiber, F-3B, and Kevlar-49 were investigated. Results show that some structure parameters - apparent crystal size, $g_{I I}$, and $f_{006}-$ are closely related to the mechanical properties of aramid fibers. It is concluded that the fiber strength and modulus is determined by combination of crystallite orientation, paracrystalline parameter and perfect degree of crystallites along fiber axis.

\section{References}

[1]. J.W.S.Hearle. High-Performance Fibres. Woodhead Publishing Ltd, 2001, p.23-53.

[2]. M.G. Northolt, R.v.d. Hout. Elastic Extension of an Oriented Crystalline Fibre. Polymer. Vol .26 (1985) No.2, 310-316.

[3]. Y. Rao, A.J. Waddon, R.J. Farris. Structure-Property Relation in Poly (P-Phenylene Terephthalamide) (PPTA) Fibers. Polymer. Vol. 42 (2001) No. 13, 5937-5946.

[4]. R. J. Young, D. Lu, R. J. Day, et al. Relationship between Structure and Mechanical Properties for Aramid Fibres[J]. Journal of Materials Science. Vol. 27 (1992) No.20, 5431-5440.

[5]. I.V. Tikhonov, A.V. Tokarev, S.V. Shorin, et al. Russian Aramid Fibres: Past-Present-Future. Fibre Chemistry. Vol. 45 (2013) No.1, 1-8.

[6]. A.A. Levchenko, E.M. Antipov, N.A. Plate, et al. Comparative Analysis of Structure and Temperature Behaviour of Two Copolyamides - Regular Kevlar and Statistical Armos. Macromolecular Symposia. Vol. 146 (1999) No.1, 145-151.

[7]. I.M. Zakharova, A.E. Zavadskii. Structural Changes in Armos Fibre as a Result of Heat Treatment in Different Conditions. Fibre Chemistry. Vol. 40 (2008) No.5, 438-440.

[8]. A.E. Zavadskii. Analysis of the Orientation of Supramolecular Structures in Fibers by the Method of X-Ray Diffractometry. Fibre Chemistry. Vol. 43 (2011) No.3, 263-266.

[9]. F.D.Wang, C.F.Chen, T.Peng, et al. Structure and properties of modified aramid fibers by benzoquinolizine heterocycle. Journal of Solid Rocket Technology. Vol. 35 (2012) No. 4, 536-540.

[10]. M.G. Northolt. X-Ray diffraction study of poly(p-phenylene terephthalamide) Fibres. European Polymer Journal.Vol.10 (1974) No. 9, 799-804.

[11]. M. Panar, P. Avakian, R.C. Blume, et al. Morphology of Poly(P-Phenylene Terephthalamide) Fibers. Journal of Polymer Science: Polymer Physics Edition. Vol.21(1983) No.10, 1955-1969. 